\title{
Tibia non-union management using limb reconstruction system fixator- a case series
}

\author{
Neetin P. Mahajan, Vaibhav V. Sakhare, Prasanna Kumar G. S.*
}

Department of Orthopedics, Grant Government Medical College, Mumbai, Maharashtra, India

Received: 08 February 2021

Revised: 13 March 2021

Accepted: 15 March 2021

\author{
*Correspondence: \\ Dr. Prasanna Kumar G. S., \\ E-mail: prasannakumargs5@gmail.com
}

Copyright: ( $)$ the author(s), publisher and licensee Medip Academy. This is an open-access article distributed under the terms of the Creative Commons Attribution Non-Commercial License, which permits unrestricted non-commercial use, distribution, and reproduction in any medium, provided the original work is properly cited.

\begin{abstract}
The management of non-unions is always the challenge to the orthopedic surgeons. It requires proper expertise in that field. Improper management leads to multiple surgeries and disability in patients. External fixation using limb reconstruction system (LRS) is one of the options in the management of these conditions. We present a series of 04 cases of non-union tibia managed with LRS fixator. All the patients had compound tibia fractures, 02 patients were managed initially with AO external fixator, one with plating and another with intramedullary nailing. Out of four cases, two cases were infected non unions and two cases were non infective non unions. All the four patients were managed secondarily with LRS fixator with less complications and good outcome. LRS fixator is one of the best options in the management of both infective and non-infective non unions of the tibia. It helps in both compression and distraction in a single frame with minimal complications. It gives good stability in the bone which helps in early weight bearing and adjacent joint range of motion.
\end{abstract}

Keywords: Tibia non-union, LRS fixator, Infection

\section{INTRODUCTION}

Fractures of the tibia are one of the commonest injuries especially with the rise in road traffic accidents. The large subcutaneous antero-medial surface predisposes to open fractures and often leads to a bone gap with devoid of soft tissue cover. Soft tissue damage and periosteal stripping are common in high velocity and open injuries, and this can compromise the vascularity of the tissues around the fracture. Infection of the wound, deformity, limb shortening and non-union are all known complications of the tibia fracture. Non-union of long bone fractures has become a common problem in orthopedic practice. Non-union of a fracture can occur both in conservative as well as in operative treatment. ${ }^{1-3}$ Infected non-union of long bones not only a source of functional disability but also it can lead to various economic and social hardship. Stability, vascularization and good rehabilitation are required for successful union of tibia fractures. Thus, the management of tibia non- union revolves around attempts at satisfactorily restoring the above-mentioned factors to bring about an adequate union for physical and psychological rehabilitation of the patient. Infected non-union can result from various etiologies, commonest being, open fractures, previous surgical procedures or as sequelae to osteomyelitis of bone.

Infected non-unions have been the challenge for orthopedic surgeons since decades, because of the following factors, i.e. a) previous surgeries would have resulted in cicatrisation of the soft tissue with an avascular environment around the fracture site, b) chronic discharging sinus suggestive of pus collection and possible presence of sequestrum, c) necrosis of fracture ends near the non-union site up to variable lengths, due to thrombosis of vascular channels of the bones, d) prolonged immobilization, multiple surgeries with fibrosis of the muscles resulting in stiffness of adjacent joints, e) the microorganism may have developed 
resistance to multiple antibiotics, f) occurrence of limb length discrepancy and deformities, and g) variable degree of soft tissue loss or defects requiring multiple sessions of plastic surgical reconstructions. ${ }^{4,5} \mathrm{We}$ present a case series of tibia non unions and their management using LRS fixator.

\section{CASE SERIES}

\section{Case 1}

A 28-year-old male presented with complaints of pain, swelling and wound $(1 \times 1 \mathrm{~cm})$ over the right knee and difficulty in walking with history of fall from bike with no distal neurovascular deficit. Patient was stabilized and Proper wound wash was given and antibiotics were started. X-rays of the tibia showed proximal tibia fracture. There was no history of diabetes or hypertension. Initially patient was operated for proximal tibia fracture with anterolateral plate. After 1 month of initial surgery, the patient came for follow up with pus discharge from sutured site. Antibiotics were started according to culture report. Patient was admitted and planned for wound debridement. After 5 months of debridement, the patient came again with discharging pus from the wound site for which implant removal was done and the LRS fixator was applied with fibular osteotomy after the debridement of the non-union site. Full weight bearing was started from the second post-operative day after the LRS fixation. The compression of the non-union site was started after one week using compression distraction (CD) devise. The regular follow-up X-rays were taken to assess the compression and union at the non-union site. At 6 months, the non-union got united without any complications and the LRS fixator was removed (Figure 1).

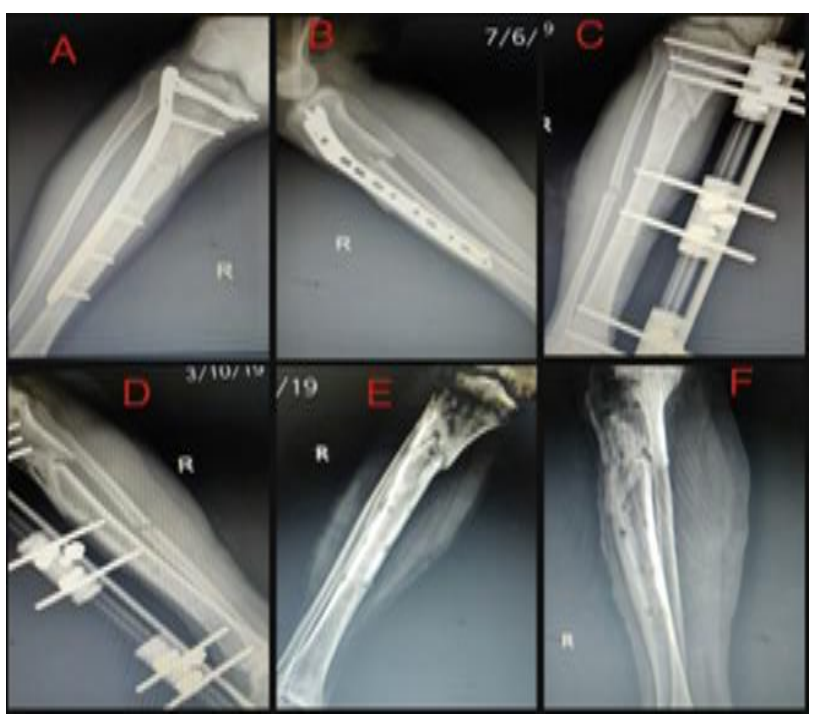

Figure 1: A, B) The anterolateral plating for proximal tibia fracture, C, D) LRS fixation after the plate removal and $E, F)$ fracture union after the $L R S$ removal.
At present one year's follow-up, the patient is having good knee and ankle range of motion with no pain and difficulty in walking.

\section{Case 2}

A 21 years old male presented with complaints of pain, swelling and wound in the left leg and difficulty in walking. Patient had a fall from bike. Patient was stabilized and proper wound wash was given and antibiotics was started. There was no history of diabetes or hypertension. On examination, there was tenderness present, and lacerated wound of $4 \times 4 \times 0.3 \mathrm{~cm}$ with no DNVD. X-ray showed left tibial fibula shaft fracture. Initial fixation was done with $\mathrm{C}$ clamp external fixator, debridement of wound was done and VAC was applied. Daily pin tract dressing and knee rom was started. After one month, skin grafting of the wound was done.

External fixator was removed after 2 months of initial surgery, on follow up after 6 months nonunion of tibia was seen in the $x$ ray with mobility at the fracture site. Patient was then admitted and operated with-LRS fixation of left tibia with primary compression without any corticotomy. Patient was mobilized with full weight bearing from second post-operative day. The non-union site was united by six months, and the fixator was removed (Figure 2). At present one and half years followup, the patient is having good knee and ankle range of motion with no pain and difficulty in walking.

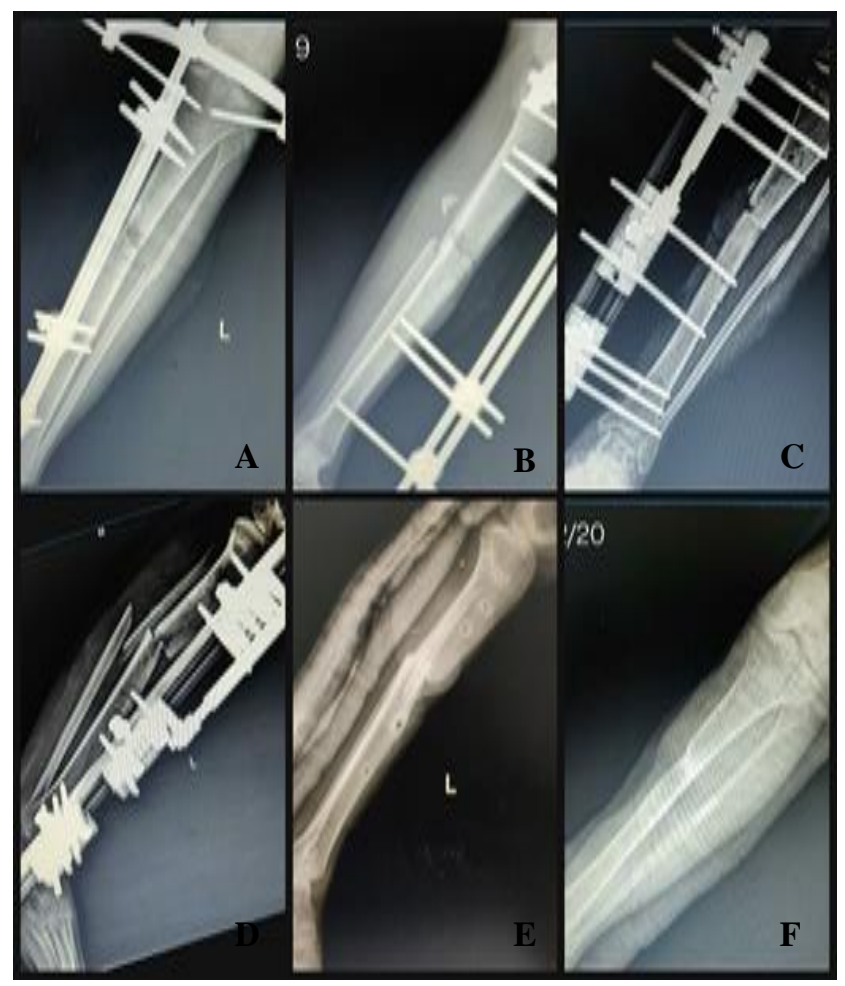

Figure 2: A, B) External fixation for compound tibia fracture, C, D) LRS fixation after external fixator removal, E, F) union after the $\mathrm{LRS}$ removal. 


\section{Case 3}

A 23 years-old male presented with complaints of pain, swelling and wound in the left leg and swelling, pain in right wrist. Patient had a fall from bicycle. Patient was stabilized and proper wound wash was given and antibiotics was started. There was no history of diabetes or hypertension. On examination, there was tenderness present at proximal tibia, wound measuring 9x6x0.8 $\mathrm{cm}$ over medial aspect of the leg below knee joint. After $\mathrm{x}$ rays of tibia, patient was diagnosed as grade $3 \mathrm{~b}$ compound fracture of left tibia fibula and right distal end radius fracture. Initially wound was debrided and fixed with C clamp external fixator and VAC was applied. Daily pin tract dressing and knee ROM was started. After 1 month skin grafting of left leg was done. External fixator was removed after 3 months. On follow up after 6 months non-union of tibia was seen in the $\mathrm{x}$ ray with mobility at the fracture site.

The non-union of the tibia was managed with LRS fixator along with fibular osteotomy without opening the nonunion site. The gradual compression of $1 \mathrm{~mm}$ /day was started after one week using compression distraction devise. The full weight bearing with knee ROM was started from the second post-operative day. Regular follow up X-rays were obtained every month till the clinical and radiological union (Figure 3). At present one year follow-up, the patient is having good knee and ankle range of motion with no pain and difficulty in walking.

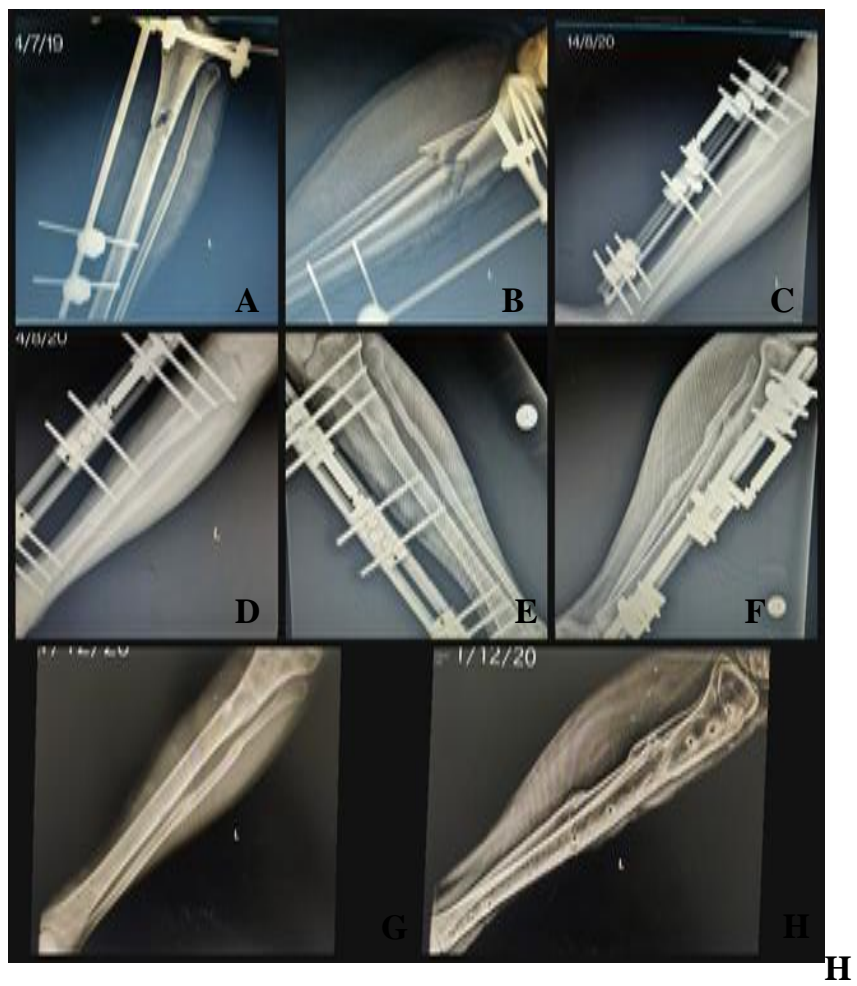

Figure 3: A, B) External fixator for tibia fracture, C, D) LRS fixation for non union, E, F) union after LRS fixation, $\mathbf{G}, \mathrm{H})$ Complete unioun after implant removal.

\section{Case 4}

A 55 years old male presented with complaints of pain, wound and difficulty in walking for six months. Patient had a history of RTA one year back and was diagnosed as compound grade 2 tibia fracture. Initial management was done using intramedullary nail in outside hospital and flap for the wound coverage. After the surgery, patient had pus discharge from the fracture site which was not relieved after the debridement and antibiotics. The intramedullary tibia nail was removed after 6 months of initial surgery because of infection and referred to our institute.

On examination, there was a wound measuring $2 \times 3 \mathrm{~cm}$ present over the medial aspect at the fracture site with pus discharge. $\mathrm{X}$ ray showed the non-union with sequestra formation. We have managed with debridement of the wound, sequestrectomy, fibular osteotomy, proximal metaphyseal tibial corticotomy and LRS fixation. Post operatively full weight bearing, knee ROM was started from the second post-operative day. The gradual distraction of $1 \mathrm{~mm} /$ day was started after one week. At around 6 weeks the distraction was stopped, follow-up $\mathrm{x}$ rays were taken every 3 weeks to assess the consolidation of the regenerate and the union of the non-union site. By 6 months, the regenerate got consolidated and non-union site showed clinical and radiological union (Figure 4). At present patient is comfortable with no pain and disability in walking.

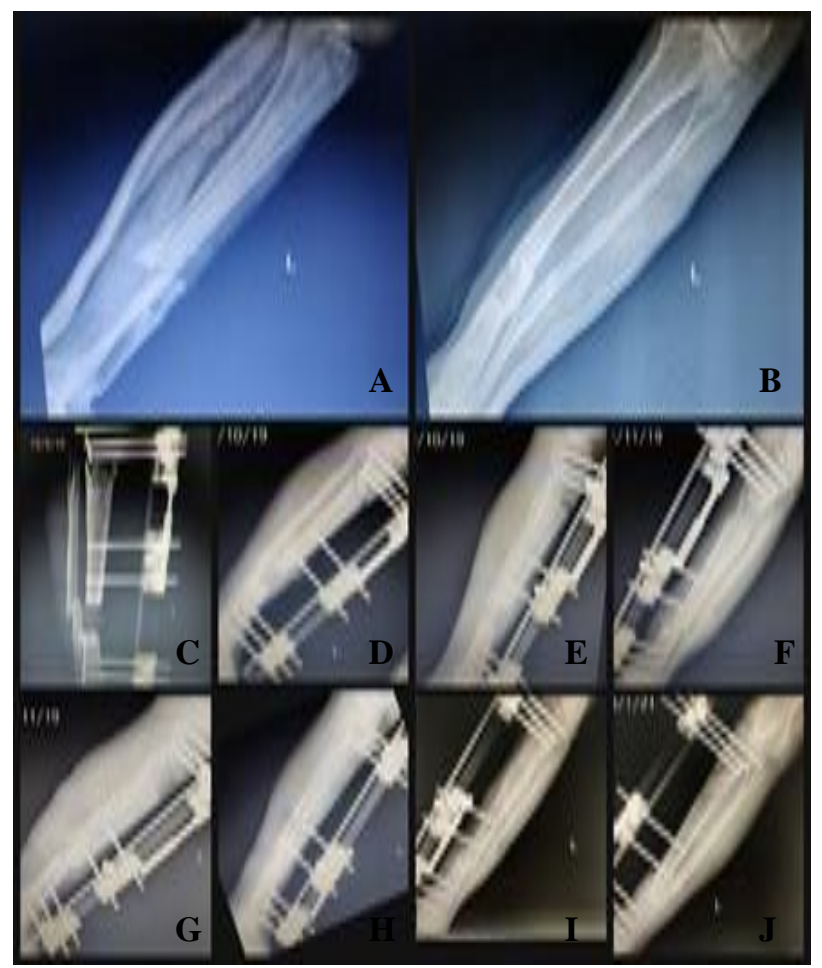

Figure 4: A, B) non union at distal third tibia, C, D) sequestrectomy and corticotomy with LRS fixation, EJ) Sequential step of compression and distraction. 


\section{DISCUSSION}

The management of non-unions is always the challenge to the orthopedic surgeons. It requires proper expertise in that field. Improper management leads to multiple surgeries and disability in patients. Non unions can be managed with both internal and external fixation, both have advantages and disadvantages. Internal fixation requires complete eradication of infection before the fixation but external fixation can be applied in cases of persistent infections also. LRS fixator is one of the simple external fixators used in tibia in the management of infective as well as non-infective non unions.

The tremendous interest has been observed in distraction osteogenesis. The clinical fact is that, the distraction can produce new bone formation. The effect of rhythmical distraction which generates new bone formation was enlightened by Illizarov. The effect of corticotomy on increased vascularity of the whole limb as well as the fixator in the fracture site was still under study.

The distraction on tensile force at the corticotomy site, the lining cells covering the bone ends are able to differentiate into osteogenic and chondrogenic cells under an adequate stimulus and environment and are called as osteosynthesis or intramembranous ossification. ${ }^{6}$ This type of regeneration of bone can be obtained by an appropriate distraction rate. This rate appears to be critical in the new bone formation and maintenance of adequate blood supply. ${ }^{7,8}$

In the present study, mono planar external fixator was used and appropriate rhythmical distraction was done. About $80 \%$ of cases showed good periosteal tube of new bone formation. Due to the preservation of medullary blood supply, the corticotomy is better than osteotomy. ${ }^{9}$ It is important to preserve the periosteum, since this layer has been demonstrated to be a most important site of osteogenesis.

The site chosen for the osteotomy should ideally, be metaphyseal or immediately sub-metaphyseal, since this is a wider and more vascular region and has been shown to have better osteogenic potential than the diaphysis. The effect of corticotomy on the healing of bone was also explained by intact intramedullary blood supply by micro angiographic studies. ${ }^{9}$

It is experimentally proved that there is no difference in regeneration to the healing sequence, in rhythmical distraction either after corticotomy or after osteotomy. The microangiographic study is essential at this juncture to prove that there is intact medullary tube after corticotomy in this series. ${ }^{9,10}$ Union achieved by repairing defects with cancellous grafts may prove to be acceptable alternatives. ${ }^{11}$ The biomechanical structure of the restored bone may require years to remodel to achieve the radiological appearance of that obtained by distraction regeneration. Recent advances in microvascular anastomosis technology have permitted vascularized osseous transfers for dealing with missing bone tissue. In the lower limb, grafts, whether fibula or iliac crest take years to hypertrophy and often fracture one or more times before complete remodelling. ${ }^{12}$ Distraction osteogenesis and bone transport can be considered to be the gold standard for infected gap non-union of the tibia as it simultaneously solves the problem of the bone gap, infection (radical debridement without fear of bone gap), deformity correction, early weight bearing and concomitant soft tissue expansion.

\section{CONCLUSION}

Limb reconstruction system (LRS) fixator is one of the best options in the management of both infective and non- infective non unions of the tibia. It helps in both compression and distraction in a single frame with minimal complications. It gives good stability in the bone which helps in early weight bearing and adjacent joint range of motion.

\section{Funding: No funding sources Conflict of interest: None declared \\ Ethical approval: Not required}

\section{REFERENCES}

1. Leiblein M, Verboket R, Marzi I, Wagner N, Nau C. Non-unions of the humerus-Treatment concepts and results of the last five years. Chinese J Traumatol. 2019;22:187-95.

2. Mills L, Tsang J, Hopper G, Keenan G, Simpson AH. The multifactorial etiology of fracture nonunion and the importance of searching for latent infection. Bone Joint Res. 2016;5:512-9.

3. Motsitsi NS. Management of infected nonunion of long bones: the last decade (1996-2006). Injury. 2008;39:155-60.

4. Rohilla R, Wadhwani J, Devgan A, Singh R, Khanna M. Prospective randomized comparison of ring versus rail fixator in infected gap nonunion of tibia treated with distraction osteogenesis. Bone Joint $\mathrm{J}$. 2016;98(10):1399-405.

5. El-Rosasy MA. Acute shortening and re-lengthening in the management of bone and soft-tissue loss in complicated fractures of the tibia. J Bone Joint Surg. 2007;89:80-8.

6. Suganthi DD, Sreekumar K. A study on application of Ilizarov ring fixator in tibial lengthening. International J Orthop Sci. 2019;5:398-400.

7. Ryan ET, Matthew JS. Skeletal blood flow in bone repair and maintenance. Bone Research. 2013;1:31122.

8. Marenzana M, Arnett TR. The key role of the blood supply to bone. Bone Res. 2013;3:203-15.

9. Dabis J, Templeton-Ward O, Lacey AE, Narayan B, Trompeter A. The history, evolution and basic science of osteotomy techniques. Strategies Trauma and Limb Reconstruct. 2017;12:169-80. 
10. Peek AC, Timms A, Chin KF, Calder P, Goodier D. Patterns of healing: a comparison of two proximal tibial osteotomy techniques. Strategies Trauma Limb Reconstruct. 2016;11:59-62.

11. Tak MW, Tak WL, Xin L, Christian F, Kelvin Y, Frankie L. Masquelet technique for treatment of post-traumatic bone defects. Sci World J. 2014;710302.
12. Singh A, Ghosh S, Chaudhuri A, Datta S, Chowdhury A, Roy DS. Ilizarov fixator in management of non-united and infected tibial shaft fractures. Med J DY Patil University. 2015;8:35-40.

Cite this article as: Mahajan NP, Sakhare VV, Prasanna GS. Tibia non-union management using limb reconstruction system fixator-a case series. Int J Res Orthop 2021;7:649-53. 